\title{
Servicios móviles de mensajería instantánea y Covid-19: oportunidades y limitaciones para la comunicación institucional
}

\section{Mobile instant messaging services and Covid-19: opportunities and limitations for institutional communication Andreu Casero-Ripollés}

Casero-Ripollés, Andreu (2019). "Servicios móviles de mensajería instantánea y Covid-19: oportunidades y limitaciones para la comunicación institucional". Anuario ThinkEPI, v. 14, e14d05.

https://doi.org/10.3145/thinkepi.2020.e14d05

Publicado en IweTel el 20 de octubre de 2020

\section{Andreu Casero-Ripollés}

https://orcid.org/0000-0001-6986-4163

Universitat Jaume I de Castelló

Departamento de Ciencias de la Comunicación

Facultad de Ciencias Humanas y Sociales

Avda. Vicent Sos Baynat, s/n. 12071 Castelló, Spain

casero@uji.es

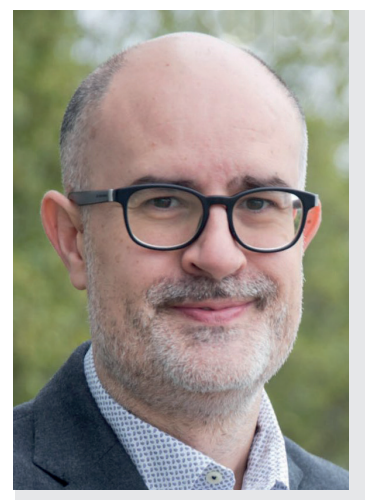

Resumen: La Covid-19 ha revelado la elevada necesidad de información que la ciudadanía experimenta en momentos críticos. Uno de los soportes clave para el acceso a la misma son los servicios móviles de mensajería instantánea, que, además, son fundamentales en las interacciones sociales en un contexto de mediatización profunda. El objetivo de este artículo es evaluar las oportunidades y limitaciones que estas plataformas han ofrecido para la comunicación institucional durante la crisis del coronavirus. Para ello, se ha explorado también el uso dado a los canales de WhatsApp y Telegram por parte de las administraciones públicas con especial atención al caso español, aunque también incorporando ejemplos internacionales. La difusión de información oficial y verificada, por un lado, y su capacidad para hacer frente a las noticias falsas, por otro, convirtieron a estos servicios en un aliado para las instituciones a la hora de prestar un servicio público de calidad en la crisis del coronavirus.

Palabras clave: Covid-19; WhatsApp; Comunicación política; Comunicación institucional; Servicios móviles de mensajería instantánea; Administraciones públicas; Gobiernos.

Abstract: The Covid-19 outbreak has revealed citizens' great need for information during times of crisis. One of the main channels for accessing information is mobile instant messaging services, which are also essential for social interactions within the context of deep mediatization. The objective of this article is to evaluate the opportunities and limitations that these platforms have offered for institutional communication during the coronavirus crisis. For this purpose, we examined the use of WhatsApp and Telegram channels by public administrations, focusing on the Spanish case in addition to incorporating other international examples. We found that the dissemination of official and verified information, on the one hand, and their ability to counter fake news, on the other, made these services a useful ally for institutions in terms of providing high-quality public service during the coronavirus crisis. 
Keywords: Covid-19; WhatsApp; Political communication; Public communication; Mobile instant messaging services; Public administrations; Governments.

\section{Introducción}

La aparición de las tecnologías digitales y la emergencia de las redes sociales han propiciado una creciente diversificación de las formas de acceso a la información y de consumo de noticias por parte de los ciudadanos. Cada vez son más las plataformas y soportes disponibles a la vez que los productores de contenido se han multiplicado. Más que un proceso de sustitución de unos medios por otros, se ha producido la generalización de una complementariedad en el consumo de medios que provoca que los ciudadanos recurran, de forma combinada, a varios medios para obtener información y enriquecer su conocimiento (Dutta-Bergman, 2004). Con ello, se ha transformado el panorama de la oferta informativa en nuestra sociedad generando oportunidades, como la variedad de fuentes informativas posibles, y también riesgos, como la sobreinformación o la desinformación.

En todo caso, este escenario está propiciando cambios en los hábitos de consumo de la información. Uno de los principales es el auge de las plataformas digitales y las redes sociales como fuente informativa. Dentro de estas, destacan particularmente los servicios móviles de mensajería instantánea como Telegram o WhatsApp. Esta última plataforma, es la que tiene un mayor número de

“Uno de los principales cambios en los hábitos de consumo de información es el auge de las plataformas digitales y las redes sociales como fuente informativa" usuarios en España en 2019 (88\%), por delante de Facebook (87\%), YouTube (68\%), Instagram (54\%) o Twitter (50\%) (IAB Spain, 2019). Además, WhatsApp recibe la mejor valoración del público (un 8,5 sobre 10) y genera un uso muy frecuente e intensivo (un $97 \%$ la utiliza diariamente). El $36 \%$ de los consumidores de noticias en línea lo emplean para informarse en 2019, situándose, así, en segunda posición solo superado por Facebook (Newman et al., 2019).

Estos datos ponen de manifiesto el papel preponderante de los servicios móviles de mensajería instantánea como soporte clave en el acceso a la información. Además, estas plataformas están ocupando un rol central en las interacciones sociales al facilitar la conexión y los intercambios entre las personas, impulsando, incluso, la participación política (Gil de Zúñiga; Ardèvol-Abreu; Casero-Ripollés, 2019). De hecho, estos servicios son uno de los principales impulsores de la mediatización profunda (Couldry; Hepp, 2018). Este proceso implica que los medios digitales se han extendido a todas las esferas sociales y se han convertido en una infraestructura indispensable para nuestra vida cotidiana. Por ello, su importancia en la escena social es relevante ya que condiciona, de forma decisiva, cómo construimos nuestro mundo y nos relacionamos con él.

La irrupción de la Covid-19 ha acentuado las tendencias en marcha en relación con el acceso y consumo de información. El brote de coronavirus ha revelado la elevada necesidad de información que la ciudadanía experimenta en momentos críticos. Los datos relacionados con las primeras semanas del confinamiento demuestran que se registró un fuerte incremento del consumo de noticias por parte del público, especialmente de los jóvenes, las personas con menor nivel educativo y los usuarios esporádicos de información (Casero-Ripollés, 2020a). Se generó un efecto de reconexión a las noticias de los públicos más alejados de las mismas, convirtiendo, así, el consumo de noticias en algo prácticamente universal. Además, el uso de WhatsApp sufrió un fuerte incremento ya que se multiplicó por ocho durante el confinamiento en España.

La información se convierte en este tipo de situaciones, como la provocada por la Covid-19, en un recurso esencial para la sociedad. En estas circunstancias, es clave para forjar confianza, generar responsabilidad y reducir la confusión y la incertidumbre. Por lo tanto, actúa como mecanismo para la articulación cívica de una comunidad de ciudadanos, por un lado, y como instrumento capaz de ofrecer orientación y conocimientos encaminados a resolver los problemas de la vida cotidiana y ser la base de los procesos de toma de decisiones de las personas, por otro. De ahí, el elevado valor social que adquiere la información en contextos como el suscitado por el coronavirus. Su difusión a tiempo real y de forma rápida pasa a ser un elemento estratégico de primer orden en la gestión de la crisis para evitar el caos, la desconfianza y las actitudes incívicas. Por ello, en estos casos, la comunicación institucional tiene que situarla en el centro de su acción.

Los gobiernos y el resto de administraciones públicas desplegaron una intensa actividad comunicativa ante la irrupción de la Covid-19 mediante estrategias para explicar las medidas adoptadas y gestionar las 
campañas de salud pública encaminadas a frenar la pandemia. En el caso de España, el Gobierno llevó a cabo una presencia comunicativa permanente, a través de diversos portavoces, y proactiva, utilizando diversos canales y soportes para establecer contacto directo con la ciudadanía (Castillo-Esparcia; Fernández-Souto; Puentes-Rivera, 2020; García-Santamaría; Pérez-Serrano; Rodríguez-Pallares, 2020). Predominó el uso intensivo de las emociones y la apelación a la unidad para generar una imagen y un mensaje positivo de naturaleza afectiva (De-Blasio; Selva, 2020). Por su parte, en las redes sociales prevaleció la difusión de información de servicio y de carácter administrativo (Castillo-Esparcia; Fernández-Souto; Puentes-Rivera, 2020). En estas plataformas, un elemento clave durante la pandemia fue el establecimiento de un diálogo con la ciudadanía (Chen et al., 2020). Con todo, estas primeras investigaciones sobre la comunicación de las instituciones públicas durante la pandemia han dedicado poca atención al análisis de los servicios móviles de mensajería instantánea. Por ello, el objetivo de este artículo es evaluar las oportunidades y limitaciones que estas plataformas, dotadas de una elevada centralidad social, han ofrecido para la comunicación institucional durante la crisis de la Covid-19. Para ello, se ha explorado también el uso dado a los canales de WhatsApp y Telegram por parte de las administraciones públicas con especial atención al caso español, aunque también incorporando ejemplos internacionales.

\section{Las prestaciones de los servicios móviles de mensajería instantánea para la comunicación institucional: oportunidades y limitaciones}

Los teléfonos inteligentes son el principal dispositivo de acceso a internet en España ya que el 96\% de los usuarios los usan para este fin (Fundación Telefónica, 2020). Por ello, se sitúan como una herramienta central en las estrategias de difusión de la información en casos de emergencia sanitaria. Una de las aplicaciones más usadas en estos terminales es la mensajería móvil instantánea. Estos servicios, entre los que sobresalen WhatsApp y Telegram, tienen una serie de potencialidades para su uso informativo. Para abordar su análisis usaremos el concepto de prestaciones o affordances. Este término, acuñado por Gibson (1979), se refiere a las posibilidades de acción que permite un objeto o aplicación tecnológica en relación con un actor social. Permite dar cuenta de la agencia tecnológica entendida como oportunidades y límites de acción producidos por las propiedades técnicas de un dispositivo o servicio en relación con las percepciones de las personas que interactúan con ellos y el contexto más amplio en que están actuando (Rice et al., 2017). Partiendo de estas coordenadas, podemos analizar el abanico de posibles acciones socio-técnicas de WhatsApp y Telegram para la comunicación institucional.

\subsection{Oportunidades}

Las investigaciones previas han identificado varias prestaciones de los servicios móviles de mensajería instantánea. Así, Klein et al. (2018) detectaron cinco grandes propiedades que permiten estas aplicaciones:

- interactividad;

- capacidad de compartir conocimiento;

- colaboración;

- ubicuidad;

- sentido de presencia.

Por su parte, Mannell (2019) estableció adicionalmente dos prestaciones relevantes:

- modulación: tiene que ver con las posibilidades de adaptar los mensajes a personas o grupos específicos;

- consumo diferido de contenidos: relacionada con el hecho que, pese a que el envío de contenidos es inmediato, estos son almacenados indefinidamente y son fáciles de recuperar para consultar en cualquier momento, ofreciendo una gran flexibilidad en el consumo de información.

Específicamente para la comunicación institucional en el contexto de la Covid-19, estos servicios móviles de mensajería instantánea presentan varias prestaciones relevantes:

- Efectividad: permiten llegar de forma directa a una gran cantidad de ciudadanos, debido a la práctica universalidad que ha adquirido el uso de algunas de estas aplicaciones como WhatsApp.

- Rapidez: posibilitan informar al público en tiempo real sobre la situación sanitaria, algo que resulta crucial en emergencias sanitarias como la Covid-19.

- En sintonía con la modulación, estos servicios permiten la personalización en la difusión de información ya que facilitan la segmentación de públicos y la adaptación de los mensajes según las características de estos. Esto último se refuerza gracias a la datificación (Couldry; Mejias, 2019), ya que 
el entorno digital favorece la obtención, acumulación y procesamiento de datos sobre intereses y preocupaciones de la ciudadanía. Esto permite trazar perfiles muy detallados de consumidores a los que se puede suministrar mensajes personalizados y adaptados, mejorando y retroalimentando el servicio de distribución de información durante el coronavirus.

- Bajo coste: es posible configurarlos de forma automatizada mediante chatbots, programas informáticos basados en la inteligencia artificial que permiten la interacción entre una máquina y un interlocutor humano agilizando, así, la difusión de información durante la Covid-19 (Chen et al., 2020).

- Favorecen la posibilidad de articular un servicio público dotado de utilidad práctica, puesto que posibilitan que la ciudadanía acceda a la información de servicio sobre temas de relevancia pública, permitiendo, así, compartir el conocimiento.

- Capacidad para combinar las dimensiones comunicativas interna (fomentando la coordinación dentro de la organización) y externa (distribuyendo contenidos hacia la ciudadanía) en una única plataforma.

Este conjunto de prestaciones o affordances permiten afirmar que los servicios móviles de mensajería instantánea resultan especialmente idóneos para la comunicación institucional, particularmente para la difusión de la información pública, especialmente de aquella relacionada con un acontecimiento prominente y crítico para toda la sociedad como la Covid-19.

\subsection{Limitaciones}

Sin embargo, estos servicios también presentan limitaciones desde el punto de vista de sus prestaciones en el contexto de la crisis del coronavirus. Una de las principales es el hecho que los ciudadanos deben ser proactivos para inscribirse por voluntad propia a este tipo de servicios. Las administraciones públicas no pueden agregar números de teléfono a sus canales de WhatsApp y Telegram sin el consentimiento expreso de los interesados para cumplir, en el caso de España, con la Ley Orgánica 3/2018, de 5 de diciembre, de Protección de datos personales y garantía de los derechos digitales (España, 2018). Esto exige realizar campañas de promoción para conseguir la adhesión de los potenciales usuarios y puede limitar el alcance de esta herramienta entre la población si no se logra la suficiente masa crítica de suscripciones.

Una segunda dificultad tiene que ver con la configuración de este tipo de canales. En términos generales, la mayoría de las administraciones públicas han optado por establecer una interactividad limitada y controlada. La capacidad de participación de los usuarios se constriñe a los cauces y acciones previamente delimitadas. Esto responde a una lógica comunicativa vertical que va de arriba, dónde se sitúa la administración pública, a abajo, dónde se ubica a los usuarios. Eso restringe las posibilidades de la ciudadanía para establecer un diálogo con las instituciones públicas y reduce la intensidad del feed-back.

Finalmente, otra limitación es que los servicios móviles de mensajería instantánea se han convertido en una de las vías preeminentes de circulación de bulos y engaños durante la Covid-19 (Casero-Ripollés, 2020b; Salaverría et al., 2020). En el caso de España, se calcula que el $80 \%$ de las noticias falsas sobre el coronavirus proceden de WhatsApp (Tardáguila, 2020). Allí somos más vulnerables porque son familiares, amigos o conocidos quienes nos envían los mensajes, hecho que activa nuestra credibilidad y nos hace bajar la guardia. Esto plantea un reto para las administraciones públicas, que pueden usar estas plataformas para combatir la desinformación, transformando una limitación en una oportunidad.

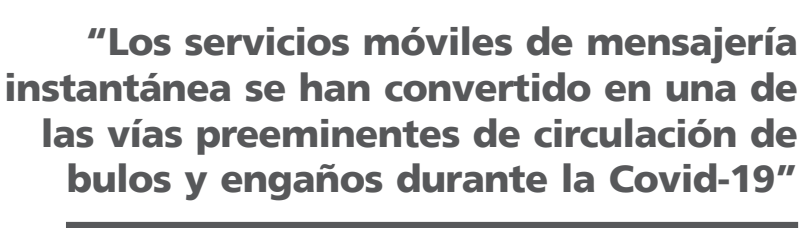

“Los servicios móviles de mensajería las vías preeminentes de circulación de bulos y engaños durante la Covid-19"

\section{El uso de los servicios móviles de mensajería instantánea por parte de las administraciones públicas en la Covid-19}

La pandemia del coronavirus ha supuesto una ocasión única para el uso de servicios móviles de mensajería instantánea por parte de las administraciones públicas. La necesidad de ofrecer información oficial, de forma rápida y directa a la ciudadanía en un contexto crítico y altamente complejo ha situado a este tipo de herramientas como uno de los canales preferentes de la comunicación institucional. Previamente, varias administraciones, principalmente de carácter local, habían ido implantando canales de WhatsApp y Telegram para informar a los ciudadanos sobre temas diversos como la oferta cultural y formativa o la actividad política de los ayuntamientos. Por ello, el uso de estos servicios en el marco de la Covid-19 se ha beneficiado, en muchos casos, de esta experiencia previa. Así, algunas administraciones públicas han adaptado sus canales para incorporar de forma preeminente contenidos sobre la pandemia. En cambio, otras han aprovechado esta coyuntura para lanzar canales nuevos y específicos para hacer frente a esta emergencia sanitaria (López-Tárraga, 2020). 
En términos generales, el empleo de este tipo de herramientas durante la crisis del coronavirus ha respondido a un doble objetivo:

- difundir información pública fiable sobre síntomas, medidas de prevención, situación de la pandemia, teléfonos de contacto o restricciones, entre otros aspectos;

- luchar contra las noticias falsas y la desinformación.

Los ejemplos son diversos, tanto en el ámbito español como a nivel internacional. La mayoría de ellos se pusieron en marcha entre finales de marzo y principios de abril de 2020, coincidiendo con una elevada afectación de la Covid-19 en todo el mundo. Además, cubrieron la totalidad de los ámbitos territoriales, desde los municipios hasta las organizaciones internacionales pasando por los Estados y las regiones o comunidades autónomas.

En España, el Gobierno del Estado, a través de la Secretaría de Estado de Digitalización e Inteligencia Artificial, promovió el Hispabot-Covid19. A través de un chatbot, este servicio ofrecía información sobre la pandemia para generar confianza entre la ciudadanía, por un lado, y para reducir la presión sobre las líneas telefónicas de atención al público, por otro. En mayo de 2020, este servicio contaba con algo más de 338.000 suscriptores (Castillo-Esparcia; Fernández-Souto; Puentes-Rivera, 2020). Asimismo, también se lanzó la aplicación Asistencia Covid-19 que permitía realizar un autodiagnóstico sobre la enfermedad y proporcionaba consejos y recomendaciones sobre cómo actuar en caso de obtener un resultado positivo.

En el ámbito regional, varias comunidades autónomas implementaron servicios móviles de mensajería instantánea relacionados con el coronavirus. Así, la Generalitat de Cataluña creó una herramienta mediante un chatbot para informar sobre la pandemia y para hacer frente a la desinformación. Igualmente, la Junta de Andalucía impulsó un canal de WhatsApp y otro de Telegram para hacer llegar la información oficial a la ciudadanía y para contribuir a la resolución de dudas.

En el terreno local, diversos ayuntamientos, como el de Almassora (Castellón), aprovecharon sus canales preexistentes para añadir información específica sobre la Covid-19, llegando a registrar, en este caso, 124 consultas durante los diez primeros días del confinamiento. Otros, como el de San Roque (Cádiz) o el de Salinas (Alicante), crearon nuevos canales específicos de WhatsApp dedicados exclusivamente a difundir información y alertas sobre la crisis sanitaria que permitían también comunicar incidencias. De esta forma, las administraciones locales explotaron la cercanía con la ciudadanía para trasladar información fiable, prestando un valioso servicio público.

Finalmente, en el ámbito internacional, la Organización Mundial de la Salud (OMS) lanzó, a finales de marzo de 2020, su servicio móvil de mensajería instantánea para difundir información sobre la Covid-19 y resolver dudas de la población sobre la enfermedad. Al igual que otros ejemplos, este canal empleó un chatbot basado en la inteligencia artificial. Los contenidos se ofrecían en 6 idiomas pudiendo llegar a un público potencial de 2.000 millones de personas. Por otra parte, en la lucha contra la desinformación, destacó el chatbot promovido por la International Fact Checking Network (IFCN) en colaboración con WhatsApp en mayo de 2020. Este servicio estaba orientado a informar sobre bulos y fomentar la verificación de datos o fact-checking. Sus contenidos permitían búsquedas por palabras clave sobre contenidos sospechosos de ser engaños, acceso a una lista actualizada de noticias falsas verificadas, consejos sobre cómo frenar la desinformación y la posibilidad de localizar agencias de verificación de datos cercanas. Todo ello, favorecía la batalla contra las noticias falsas en uno de los canales donde más proliferaron las mentiras durante el brote de coronavirus.

\section{Conclusiones: un servicio público estratégico en la lucha contra la Covid-19}

Los servicios móviles de mensajería instantánea se configuraron durante la Covid-19 como un aliado de las administraciones públicas para prestar un servicio público de calidad. El uso de canales de WhatsApp o Telegram por parte de organismos internacionales, gobiernos o ayuntamientos se presentó como una oportunidad estratégica y permitió ofrecer información confiable, un ingrediente que constituye una de las bases de la democracia, especialmente en momentos críticos. Además, con ello se contribuyó a combatir contra la desinformación, que conlleva numerosos peligros en situaciones de alerta sanitaria. La difusión de información oficial y verificada, por un lado, y su capacidad para hacer frente a las noticias falsas, por otro, convirtieron a estos servicios en un instrumento útil en la crisis “La difusión de información oficial y verificada, por un lado, y su capacidad para hacer frente a las noticias falsas, por otro, convirtieron a estos servicios en un instrumento útil en la crisis del coronavirus" del coronavirus. 
La implementación de estos canales durante la Covid-19 ha abierto oportunidades para las instituciones públicas que han podido tener efectos positivos. Entre ellos, destaca la posibilidad de ganar legitimidad y credibilidad en un contexto marcado por la crisis de la política tradicional y de las fuentes oficiales. El recurso a los servicios móviles de mensajería instantánea, una herramienta plenamente incorporada en las dinámicas cotidianas de millones de personas, ha podido servir para reivindicar el papel de las administraciones públicas como instancia confiable en el terreno informativo durante la pandemia. Asimismo, también ha podido favorecer una mayor conexión con la ciudadanía en una situación crítica, especialmente en el ámbito local. Esto ha podido redundar en un fortalecimiento de los vínculos comunitarios y en una mejora de la participación política, algo que WhatsApp fomenta en diversos grupos de edad, según investigaciones recientes (Gil de Zúñiga; Ardèvol-Abreu; Casero-Ripollés, 2019).

Con todo, el empleo de servicios móviles de mensajería instantánea se ha revelado como un instrumento beneficioso para la comunicación institucional durante la Covid-19. Pese a ello, su uso también ha presentado limitaciones como la reducida interactividad y la necesidad de aumentar la segmentación de los mensajes en función de las características de los usuarios (Castillo-Esparcia; Fernández-Souto; Puentes-Rivera, 2020). Sin embargo, es probable que uno de los efectos a corto plazo del empleo de estos servicios, y de las oportunidades que aportan, sea el incremento del número de administraciones públicas que pongan en marcha este tipo de herramientas, fomentando la multiplicación de canales y la diversificación de contenidos en circulación, especialmente en tiempos de crisis.

\section{Referencias}

Casero-Ripollés, Andreu (2020a). "Impact of Covid-19 on the media system. Communicative and democratic consequences of news consumption during the outbreak". El profesional de la información, v. 29, n. 2, e290223. https://doi.org/10.3145/epi.2020.mar.23

Casero-Ripollés, Andreu (2020b). "La Covid-19 en el periodismo: un impacto ambivalente". Revista de la Asociación Española de Investigación de la Comunicación, v. 7, n. 14, pp. 2-26.

https://doi.org/10.24137/raeic.7.14.1

Castillo-Esparcia, Antonio; Fernández-Souto, Ana-Belén; Puentes-Rivera, Iván (2020). “Comunicación política y Covid-19. Estrategias del Gobierno de España". Profesional de la información, v. 29, n. 4, e290419. https://doi.org/10.3145/epi.2020.jul.19

Chen, Qiang; Min, Chen; Zhang, Wei; Wang, Ge; Ma, Xiaoyue; Evans, Richard (2020). "Unpacking the black box: How to promote citizen engagement through government social media during the Covid-19 crisis". Computers in human behavior, v. 110, 106380.

https://doi.org/10.1016/j.chb.2020.106380

Couldry, Nick; Hepp, Andreas (2018). The mediated construction of reality. Cambridge: Polity. ISBN: 9780745681306

Couldry, Nick; Mejias, Ulises A. (2019). The costs of connection: How data is colonizing human life and appropriating it for capitalism. Stanford: Stanford University Press. ISBN: 9781503603660

De-Blasio, Emiliana; Selva, Donatella (2020). "Affective governance during the Covid-19 crisis: Building leadership, trust, and good citizens". Trípodos, v. 1, n. 47, pp. 67-86.

Dutta-Bergman, Mohan J. (2004). "Complementarity in consumption of news types across traditional and new media". Journal of broadcasting \& electronic media, v. 48, n. 1, pp. 41-60.

https://doi.org/10.1207/s15506878jobem4801_3

España (2018). "Ley Orgánica 3/2018, de 5 de diciembre, de Protección de datos personales y garantía de los derechos digitales". BOE, n. 294, 6 diciembre.

https://www.boe.es/buscar/doc.php?id=BOE-A-2018-16673

Fundación Telefónica (2020). Sociedad digital en España 2019. Madrid: Taurus.

García-Santamaría, José-Vicente; Pérez-Serrano, María-José; Rodríguez-Pallares, Miriam (2020). "Portavoces oficiales y estrategia audiovisual en la crisis de la Covid-19 en España". Profesional de la información, v. 29, n. 5, e290513.

https://doi.org/10.3145/epi.2020.sep.13

Gibson, James J. (1979). The ecological approach to visual perception. Boston: Houghton Mifflin. ISBN: 978 0898599596

Gil de Zúñiga, Homero; Ardèvol-Abreu, Alberto; Casero-Ripollés, Andreu (2019). "WhatsApp political discussion, conventional participation and activism: exploring direct, indirect and generational effects". Information, communication \& society, first online.

https://doi.org/10.1080/1369118X.2019.1642933

IAB Spain (2019). Estudio anual de redes sociales 2019. IAB Spain.

https://iabspain.es/wp-content/uploads/2019/06/estudio-anual-redes-sociales-iab-spain-2019_vreducida.pdf 
Klein, Amarolinda Z.; Junior, José-Carlos-da-Silva-Freitas; Da Silva, Juliana-Vitória-Vieira-Matiello-MatieIlo; Barbosa, Jorge-Luis-Vitoria; Baldasso, Lucas (2018). "The educational affordances of mobile instant messaging (MIM): Results of Whatsapp ${ }^{\circledR}$ used in higher education". International journal of distance education technologies, v. 16, n. 2, pp. 51-64.

https://doi.org/10.4018/IJDET.2018040104

López-Tárraga, Ana-Belén (2020). “Comunicación de crisis y Ayuntamientos: el papel de Telegram durante la crisis sanitaria de la Covid-19". Revista de la Asociación Española de Investigación de la Comunicación, v. 7, n. 14, pp. 104-126. https://doi.org/10.24137/raeic.7.14.5

Mannell, Kate (2019). "A typology of mobile messaging's disconnective affordances". Mobile media \& communication, v. 7, n. 1, pp. 76-93.

https://doi.org/10.1177/2050157918772864

Newman, Nic; Fletcher, Richard; Kalogeropoulos, Antonis; Nielsen, Rasmus-Kleis (2019). Reuters Institute digital news report 2019. Oxford: Reuters Institute for the Study of Journalism.

https://reutersinstitute.politics.ox.ac.uk/sites/default/files/inline-files/DNR_2019_FINAL.pdf

Rice, Ronald E.; Evans, Sandra K.; Pearce, Katy E.; Sivunen, Anu; Vitak, Jessica; Treem, Jeffrey W. (2017). "Organizational media affordances: Operationalization and associations with media use". Journal of communication, v. 67, n. 1, pp. 106-130.

https://doi.org/10.1111/jcom.12273

Salaverría, Ramón; Buslón, Nataly; López-Pan, Fernando; León, Bienvenido; López-Goñi, Ignacio; Erviti, María-Carmen (2020). "Desinformación en tiempos de pandemia: tipología de los bulos sobre la Covid-19". Profesional de la información, v. 29, n. 3, e290315.

https://doi.org/10.3145/epi.2020.may.15

Tardáguila, Cristina (2020). "The demand for Covid-19 facts on WhatsApp is skyrocketing". Poynter, 26 marzo. https://www.poynter.org/fact-checking/2020/the-demand-for-covid-19-facts-on-whatsapp-is-skyrocketing/

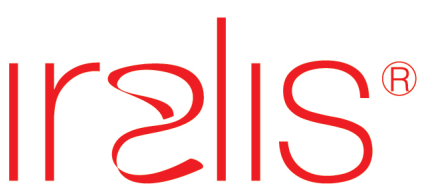

International Registry for Authors: Links to Identify Scientists

es:

- una guía para los autores hispanos para que firmen sus trabajos en el formato internacional usual

- una base de datos que registra las variantes de firma usadas por cada autor en diferentes épocas

- un buscador que usa automáticamente todas las variantes registradas

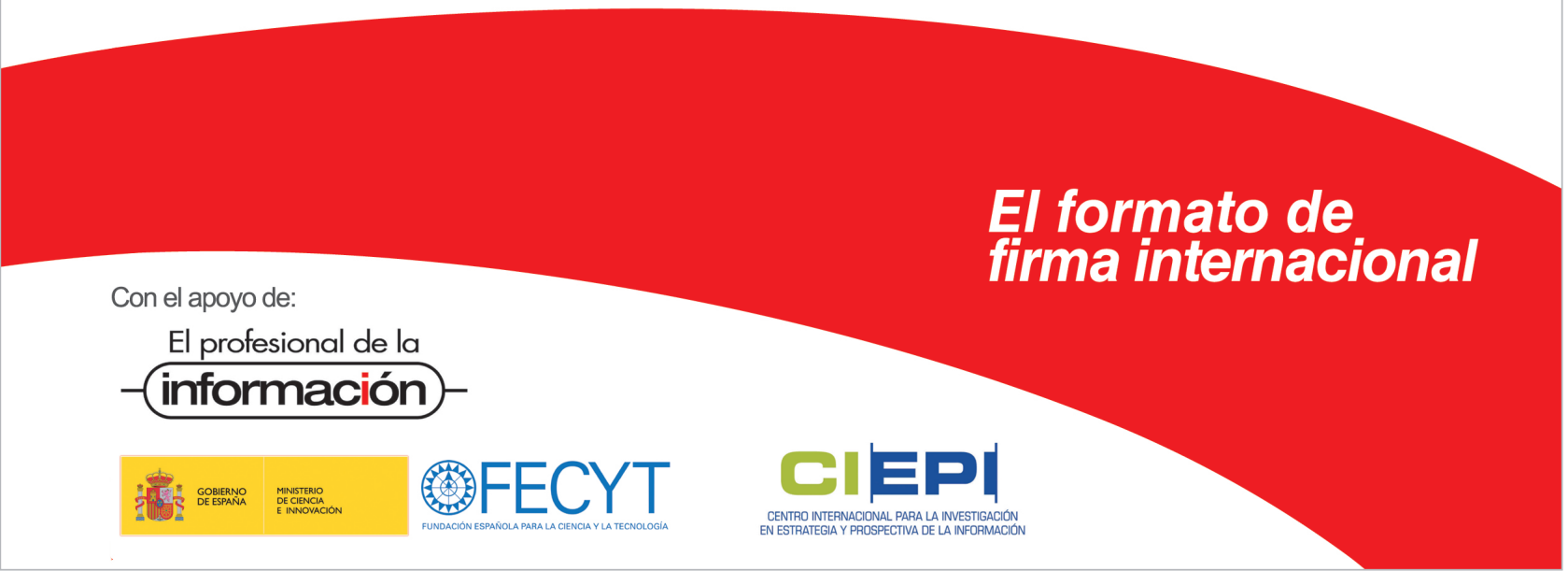

